\title{
Family Medicine's Task in Population Health: Defining It and Owning It
}

Joyce Hollander-Rodriguez, MD; Jennifer E. DeVoe, MD, DPhil

(Fam Med. 2018;50(9):659-61.)

doi: 10.22454/FamMed.2018.868771

A s family physicians we are trained to approach every patient in the context of family, and every family in the context of community. Working to improve the health of communities we serve is not a new concept, yet we continue to struggle with this crucial task for two key reasons: we let others define the terms, and we lack agreement on our role.

Family physicians have difficulty defining population health due to debates about whether this concept is new or old, who we want to include in the population, and the most effective approaches to improving a population's health. Large health systems, payers, accountable care organizations, policy makers, patients, and the public all mean different things when we talk about population health. There are distinctions in meaning between the terms population health management, public health, community health, and clinical population medicine. The terminology matters because it determines who is in the population served and who works to improve the health of that group. As family physicians and family medicine educators we must take charge of these definitions, or subsets of our patients and our communities will be left behind.

As the specialty that views every patient in the context of family and community, we should be leading population health efforts. But family physicians have difficulty being accountable for population health because it sometimes feels threatening to the patientphysician dyad: if we embrace population health initiatives, are we abandoning relationships with individual patients? With our history of community orientation, new calls for population health sound a lot like revisiting old ideas about how to do community-oriented primary care (COPC). ${ }^{1,2}$ While it is important to recognize the significant overlap with these historical approaches, we must also acknowledge that none of these approaches were widely implemented. The dominant paradigm of today's health care system has been largely shaped by market forces, but efforts to curtail health care spending have brought payers and others back to an interest in population health, and this creates a new window of opportunity. Payers are interested in population health with a narrow definition that includes members on their plan, just as health systems and clinics are likely to include only those accessing care or on patient panels. As we seize this opportunity to participate in conversations around population health, we have an ethical imperative to lead these conversations in a direction that will not exclude vulnerable subpopulations. Family physicians are uniquely positioned to advocate for population health using both narrow and broad definitions.

\section{Population Health Is Clinical Population Medicine}

By practicing clinical population medicine and embracing the "conscientious, explicit and judicious application of population health approaches to care for individual patients and to design health care systems," we can more effectively shift from reactive to proactive care. ${ }^{3}$ For example, we can design payment systems

From the Oregon Health \& Science University Department of Family Medicine, Portland, OR. 
that shift away from retrospective payments for visits toward prospective payments for populations. We can create metrics based on comprehensive health measures rather than disease-specific categories. Changing the paradigm from treating diseases to healing people can help to address the paradox of primary care and support systems to improve health for populations. ${ }^{4}$

As we launch new clinical population medicine efforts, there are several tools and skills to develop, and new team members to recruit and deploy. We can continue utilizing data to drive quality improvement decisions, to inform tracking systems, and to measure the care we provide. ${ }^{5}$ We can accelerate the implementation of enhanced primary care delivery models. We can partner with informaticists, epidemiologists, and biostatisticians to create new methods for harnessing and analyzing data from electronic health records (EHRs), insurance claims, and public health data repositories to better understand our population health landscape and how we might be able to impact it. We can identify and address social determinants of health (SDH) through the collection of individual-level SDH information, the integration of community-level SDH data (community vital signs), and develop new systems of care that incorporate referrals to community services known to improve health. ${ }^{6-8}$

\section{Population Health Is Also Community- Oriented Primary Care}

Some of the most vulnerable people in our communities do not have a medical home. ${ }^{9}$ While improving our skills in clinical population medicine, we must simultaneously work to address the needs of our broader community and reach the populations that are not regularly accessing primary care. When we define population health as the community's health, our approach is different. The Folsom Report ${ }^{10}$ of 1967 urged primary care physicians to address community health needs by partnering with public health systems and others to create communities of solution that cross jurisdictional lines. ${ }^{11}$ Despite the efforts of community-oriented primary care providers, many of the market forces at work in the US health care system led to the fragmentation we now see. Family Medicine for America's Health has helped reinvigorate this call to action and create a new vision for the role of primary care in redesigning health care and addressing the health of populations. ${ }^{12}$ We should be partnering with everyone and anyone who works to improve the health of groups of people: hospitals/health systems, payers, other primary care providers, the public health system, educational institutions, community organizations, and researchers.

Equipping the next generation of family physicians and primary care teams to be experts in population health will require many of the unique skills already taught in family medicine residencies for understanding and addressing biopsychosocial factors impacting health. We should consider adding several additional skills to our training program curricula: community engagement, patient empowerment, community organizing, collaboration and teamwork, relational leadership, informatics, data analysis, and creative problem solving (to name a few).

\section{Tangible Next Steps for Family Medicine Educators}

Teaching residents and faculty clinicians to generate reports from their EHRs about their practice panel remains an important skill, but it is equally important for them to learn how to respond to these reports. Further, we can develop and test new ways to use EHRs to prioritize care, improve risk stratification, and incorporate community vital signs into our patient care and population health efforts. ${ }^{7,8} \mathrm{We}$ can equip learners with skills for conducting community health needs assessments and for identifying adverse SDH for our patient populations. We can remind our teams that every point in a data set is a person, and that one antidote for frustration in practice is to effect change at the systems level.

Advocacy work and involvement in community organizations are important components of resident and faculty time. We identified a need to shift our approach from viewing this as an optional extracurricular activity to recognizing it as essential. Many family medicine faculty sit on community boards, lead community advisory groups, work as public health officers, and engage as advocates on issues addressing health; we need to incorporate and recognize this work as part of our role as family medicine educators and train residents in this same model. 


\section{Conclusion}

Working to address the tension between taking care of the individual patient and the magnitude of improving the health of a whole community will always be central to our discipline. The increased spotlight on this topic from payers, health systems, researchers and others suggests that we have the potential to build better teams so we are not trying to address these issues alone. Let's stop arguing about the definition of population health and take the lead on programs to improve the health of populations and communities. Let's stop feeling as if we need to choose between incorporating clinical population medicine versus community-oriented primary care into our curriculum - we need both. The population is our newest patient.

ACKNOWLEDGMENTS: The authors thank the faculty and residents at the OHSU Cascades East Family Medicine Residency with whom they had discussions that inspired this article. Special thanks to Amanda Delzer Hill, OHSU Department of Family Medicine Publications Coordinator, for her help with manuscript preparation.

CORRESPONDENCE: Address correspondence to Dr Joyce Hollander-Rodriguez, Oregon Health \& Science UniversityFamily Medicine, Cascades East Residency, 2801 Daggett Ave, Klamath Falls, OR 97601. hollajoy@ohsu.edu.

\section{References}

1. Longlett SK, Kruse JE, Wesley RM. Community-oriented primary care: historical perspective. J Am Board Fam Pract. 2001;14(1):54-63.
2. Nutting PA, ed. Community-Oriented Primary Care: From Principal to Practice. Albuquerque, NM: University of New Mexico Press; 1987.

3. Orkin AM, Bharmal A, Cram J, Kouyoumdjian FG, Pinto AD, Upshur R. Clinical population medicine: integrating clinical medicine and population health in practice. Ann Fam Med. 2017;15(5):405-409.

4. Stange KC, Ferrer RL. The paradox of primary care. Ann Fam Med. 2009;7(4):293-299.

5. Fields SA, Cohen D. Performance enhancement using a balanced scorecard in a Patient-centered Medical Home. Fam Med. 2011;43(10):735-739.

6. DeVoe JE, Bazemore AW, Cottrell EK, et al. Perspectives in primary care: a conceptual framework and path for integrating social determinants of health into primary care practice. Ann Fam Med. 2016;14(2):104-108.

7. Hughes LS, Phillips RL Jr, DeVoe JE, Bazemore AW. Community vital signs: taking the pulse of the community while caring for patients. J Am Board Fam Med. 2016;29(3):419422.

8. Bazemore AW, Cottrell EK, Gold R, et al. "Community vital signs": incorporating geocoded social determinants into electronic records to promote patient and population health. J Am Med Inform Assoc. 2016;23(2):407-412.

9. Sharfstein JM. The strange journey of population health Milbank Q. 2014;92(4):640-643.

10. National Commission on Community Health Services (NCCHS). Health is a Community Affair-Report of the National Commission on Community Health Services (NCCHS). Cambridge, MA: Harvard University Press; 1967.

11. Folsom Group. Communities of solution: the Folsom Report revisited. Ann Fam Med. 2012;10(3):250-260.

12. Puffer JC, Borkan J, DeVoe JE, et al. Envisioning a new health care system for America. Fam Med. 2015;47(8):598603. 